\title{
Multi-pair Two-way Relay Channel with Multiple Antenna Relay Station
}

\author{
Erhan Yilmaz, Randa Zakhour, David Gesbert and Raymond Knopp \\ EURECOM \\ 06560 Sophia-Antipolis, France \\ Email: \{yilmaz, zakhour, gesbert, knopp\}@eurecom.fr
}

\begin{abstract}
We consider a multi-pair two-way relay channel (TWRC) where the single-antenna mobile terminals (MT) on each pair seek to communicate, and can do so, via a common multiple antenna relay station (RS). In the multi-pair TWRC, the main bottleneck on system performance is the interference seen by each MT due to the other communicating MT pairs. In this paper, we try to tackle this problem in the spatial domain by using multiple antennas at the RS. Considering Amplify-and-Forward (AF) and Quantize-and-Forward $(\mathrm{QF})$ relaying strategies, different transmit/receive beamforming schemes at the $\mathrm{RS}$ are proposed. We compare our proposed schemes to each other and to the Decode-and-Forward (DF) relaying strategy with achievable sumrate taken as a performance metric and show that in a wide range of signal-to-noise ratio (SNR) our schemes outperform the DF relaying strategy.
\end{abstract}

\section{INTRODUCTION}

In the last decade, a great deal of research has focused on the relay channel which has found applications in cellular, mobile ad-hoc and sensor networks due to the potential improvements in system performance provided by the relaying mechanism such as reducing deployment cost, enhancing network capacity, extending radio range, mitigating shadowing effect and providing spatial diversity [2], [3]. Different relaying strategies have been proposed to improve spectral efficiency and system performance. Among these amplify-and-forward (AF), decode-and-forward (DF) and compress/Quantize-andforward (CF/QF) [1]-[3] are the most studied.

In this paper, we consider a practical half-duplex operation at the mobile terminals (MTs) and the relay station (RS). Although providing the advantages specified above, the halfduplex constraint at the RS imposes a well-known pre-log factor $1 / 2$ for the overall system throughput and therefore limits the achievable spectral efficiency. To circumvent the spectral efficiency loss in the one-way relay channel the two-way relay channel (TWRC) has recently been proposed: here both nodes exchange information via the intermediate RS [4]-[11]: this kind of scenario can occur in satellite communications or in public safety networks for example [5]. The MTs send their messages to the RS, which then processes the received signals according to a given relaying strategy and broadcasts to the MTs. This two-way relaying provides interference-free reception since at each MT the selfinterference can be canceled before decoding the unknown message. For the TWRC, analog network coding where the mobiles' signals are combined in the air and digital network coding where the RS first decodes the mobiles' signals and combines the decoded bits using the bit-wise XOR operation are the main schemes considered. [12], [13] study the single pair TWRC with multiple antenna RS and propose different RS precoding schemes.

The two-pair TWRC can be generalized to the case where more bi-directional pairs wish to communicate with each other via a single RS [14]-[16], which is the setup we consider here. Both [14] and [15] deal with a multi-pair multi-antenna RS TWRC with DF relaying followed by digital network coding (bitwise XOR) scheme: In [14], a precoding matrix optimization algorithm is developed for maximizing the sumrate of the system, whereas [15] propose a multi-group multicast aware beamforming scheme for the transmission in the second phase. Both separate MTs spatially using a multiantenna RS. In [16], on the other hand, a single antenna RS orthogonalizes the users in the studied multi-pair TWRC using Code Division Multiple Access.

In this paper, we consider a multi-pair TWRC with a multiantenna RS and focus on $\mathrm{AF}$ and $\mathrm{QF}$ relaying strategies: these are particularly attractive when there is a complexity constraint at the relay node or when the latter is oblivious to the codebooks of the MTs, in which case DF would not be possible. Moreover, as our simulations will show there are SNR ranges over which they outperform the DF strategy. We thus propose specific schemes for both types of relaying and analyze their achievable sum-rate performance. In particular, two beamforming schemes are proposed for AF relaying: a simple Tx-Rx zero-forcing (ZF) scheme and a Tx-Rx blockdiagonalization (BD)-based scheme, adapted to our specific setup. Then, for QF based relaying, we let the RS separate the signals corresponding to each pair and quantize the processed received signals as accurately as allowed by the achievable rates in the second hop of communication. Here, by taking into account the side information at each MT, we quantize a scalar which is an appropriately selected linear combination of the processed RS received signal vector, thereby avoiding vector quantization. This approach may be seen as an analog form of network coding.

\section{System Model}

The communication scenario considered is depicted in Fig.1 where $K$ pairs of single-antenna nodes (mobiles) each communicate bi-directionally via a single $M$-antenna relay; there 


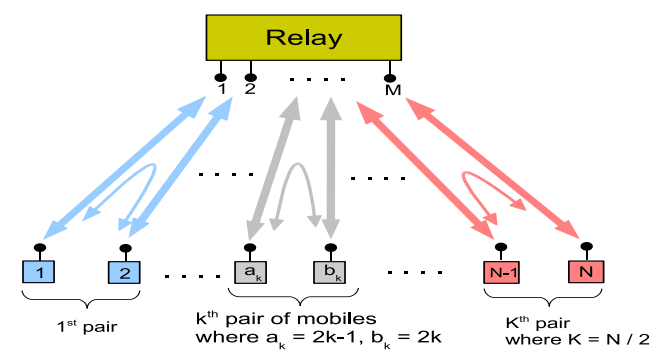

Fig. 1. $K$-pair ( $N=2 K \mathrm{MTs})$ TWRC with an $M$ antenna RS.

are no direct links between any of the $N=2 K$ single antenna mobiles, only between the nodes and the relay, as is standardly assumed, e.g. in [5], [12], [14]. Transmission is divided into two phases with equal time duration. In the first phase, the nodes simultaneously transmit their messages to the RS (uplink communication from the MTs to the RS); in this phase, as in [14], channel state information (CSI) is not available at the MTs but is available at the RS. In the second phase, the RS transmits some processed version of the received signal (downlink communication from the RS to the MTs); here CSI is available at both the transmitting RS and the receiving MTs. The exact relaying schemes used will be detailed in the next two sections.

Each node knows its own signal and can thus cancel it out of the downlink transmission, and since it only needs one of the other messages, the remaining $N-2$ messages will thus constitute interference. This is different (due to the side information available at each receiver) but could be related to a MIMO broadcast channel.

Denote by $\mathbf{h}_{i}$ the channel coefficients vector between node $i$ and the relay in the uplink direction, $\mathbf{g}_{i}$ the corresponding channel in the downlink direction; both $\mathbf{h}_{i}$ and $\mathbf{g}_{i} \in \mathbb{C}^{M}$. Further, let the $k$-th communicating pair consist of mobile $a_{k}$ and mobile $b_{k}$ where $a_{k}=2 k-1$ and $b_{k}=2 k, k=1, \ldots, K$.

The signal received at the RS in the first phase is given by:

$$
\mathbf{y}_{R}=\sum_{i=1}^{N} \mathbf{h}_{i} x_{i}+\mathbf{n}_{R}
$$

where $x_{i}$ is the signal transmitted from mobile $i$, and is subject to an average power constraint $p_{i}:=\mathbb{E}\left|x_{i}\right|^{2}$, for $i=1, \ldots, N$. The noise vector $\mathbf{n}_{R}$ is assumed to have independent identically distributed components which are zeromean complex circularly symmetric Gaussian variables of variance $\sigma_{R}^{2}, \mathcal{C N}\left(0, \sigma_{R}^{2}\right)$. Similarly, the signal received at MT $i$ in the second phase is given by:

$$
y_{i}=\mathbf{g}_{i}^{T} \mathbf{x}_{R}+n_{i}
$$

where $\mathbf{x}_{R} \in \mathbb{C}^{M}$ is the relay transmit signal and is subject to an average power constraint $P_{R}$. The noise $n_{i}$ is $\mathcal{C N}\left(0, \sigma^{2}\right)$.

Our main performance measure is the overall sum-rate:

$$
R_{\text {sum }}=\frac{1}{2} \sum_{i=1}^{N} \log _{2}\left(1+\gamma_{i}\right)
$$

where $1 / 2$ is due to the half-duplex relaying and $\gamma_{i}$ is the receive SNR (or SINR) at node $i$. The $\gamma_{i}$ 's, as well as the signals transmitted depend on the exact relay strategy adopted, and are specified in the next two sections.

\section{AMPLIFY-AND-FORWARD (AF) RELAYING SCHEMES}

In AF relaying, the RS transmit signal, $\mathbf{x}_{R}$, is given by

$$
\mathbf{x}_{R}=\mathbf{A}_{R} \mathbf{y}_{R}
$$

where the linear processing matrix $\mathbf{A}_{R} \in \mathbb{C}^{M \times M}$ is selected so as to meet the RS power constraint:

$$
\mathbb{E}\left[\mathbf{x}_{R}^{H} \mathbf{x}_{R}\right]=\operatorname{Tr}\left[\left(\sum_{i=1}^{N} p_{i} \mathbf{h}_{i} \mathbf{h}_{i}^{H}+\sigma^{2} \mathbf{I}\right) \mathbf{A}_{R}^{H} \mathbf{A}_{R}\right] \leq P_{R}
$$

After eliminating its own signal (recall that we assume CSIR), the SINR at MT $a_{k}, \forall k$ will be given by:

$$
\gamma_{a_{k}}=\frac{p_{b_{k}}\left|\mathbf{g}_{a_{k}}^{T} \mathbf{A}_{R} \mathbf{h}_{b_{k}}\right|^{2}}{\sigma^{2}+\sigma_{R}^{2}\left\|\mathbf{g}_{a_{k}}^{T} \mathbf{A}_{R}\right\|^{2}+\sum_{j \neq a_{k}, b_{k}} p_{j}\left|\mathbf{g}_{a_{k}}^{T} \mathbf{A}_{R} \mathbf{h}_{j}\right|^{2}} .
$$

\section{A. General structure of the linear processing matrix $\boldsymbol{A}_{R}$}

The structure of the linear processing matrix at the RS, $\mathbf{A}_{R}$, has yet to be specified. The optimal structure given our performance metric would maximize the sum rate subject to the given power constraint at the relay. To avoid a non-tractable optimization problem, we instead resort to suboptimal structures, which ensure that inter-pair interference is eliminated. To guarantee the feasibility of such a solution, we assume that $M \geq N=2 K$. Moreover, to formulate the proposed suboptimal solutions, we find it useful to decompose $\mathbf{A}_{R}$ into:

$$
\mathbf{A}_{R}=\mathbf{M} \mathbf{D} \mathbf{F}
$$

where $\mathbf{M} \in \mathbb{C}^{M \times \bar{R}}, \mathbf{D} \in \mathbb{C}^{\bar{R} \times \bar{L}}$ and $\mathbf{F} \in \mathbb{C}^{\bar{L} \times M},(\bar{R}$ and $\bar{L}$ will be specified later) and have the following forms:

$$
\begin{aligned}
\mathbf{M} & =\left[\begin{array}{llll}
\mathbf{M}_{1} & \mathbf{M}_{2} & \ldots & \mathbf{M}_{K}
\end{array}\right], \\
\mathbf{D} & =\operatorname{diag}\left\{\begin{array}{llll}
\mathbf{D}_{1}, & \mathbf{D}_{2}, \ldots, \mathbf{D}_{K}
\end{array}\right\}, \\
\mathbf{F} & =\left[\begin{array}{llll}
\mathbf{F}_{1}^{T} & \mathbf{F}_{2}^{T} & \ldots & \mathbf{F}_{K}^{T}
\end{array}\right]^{T},
\end{aligned}
$$

where $\mathbf{M}_{k} \in \mathbb{C}^{M \times \bar{R}_{k}}, \mathbf{D}_{k} \in \mathbb{C}^{\bar{R}_{k} \times \bar{L}_{k}}$ and $\mathbf{F}_{k} \in \mathbb{C}^{\bar{L}_{k} \times M}$, $k=1,2, \ldots, K$. Thus $\bar{R}=\sum_{k=1}^{K} \bar{R}_{k}$ and $\bar{L}=\sum_{k=1}^{K} \bar{L}_{k}$ where $\bar{R}_{k}$ and $\bar{L}_{k}$ are specified later. Letting $\mathbf{A}_{k}=\mathbf{M}_{k} \mathbf{D}_{k} \mathbf{F}_{k}$ :

$$
\mathbf{A}_{R}=\sum_{k=1}^{K} \mathbf{A}_{k}
$$

\section{B. Receive and Transmit Zero-Forcing at the Relay}

The simplest RS precoding scheme which satisfies the no inter-pair interference constraint we have imposed is one that only allows its intended signal to reach each receiver (thus even its own transmitted signal is canceled out by the RS). In the first hop the RS implements the well-known ZF receive filter then it permutes the signal position so as to ensure that each signal arrives at its destination. After that it uses a ZF 
transmit filter to send the signals to the MTs in the second hop.

The $\mathrm{ZF}$ receive filter is given by:

$$
\mathbf{F}=\mathbf{H}^{\dagger}=\left(\mathbf{H}^{H} \mathbf{H}\right)^{-1} \mathbf{H}^{H} .
$$

Then, the RS permutes the received signals with the following matrix:

$$
\underline{\mathbf{D}}=\operatorname{diag}\left(\underline{\mathbf{D}}_{1}, \ldots, \underline{\mathbf{D}}_{K}\right) \quad \text { where } \quad \underline{\mathbf{D}}_{k}=\left[\begin{array}{cc}
0 & 1 \\
1 & 0
\end{array}\right], \forall k
$$

and we define $\mathbf{D}=\rho \underline{\mathbf{D}}$ where $\rho$ is for transmit power scaling. For transmission the RS chooses the following ZF transmit filter:

$$
\mathbf{M}=\mathbf{G}^{\dagger}=\mathbf{G}^{H}\left(\mathbf{G G}^{H}\right)^{-1}
$$

and the corresponding RS precoding matrix is given by:

$$
\mathbf{A}_{R}=\mathbf{M D F}=\rho \mathbf{M D} \underline{\mathbf{F}}=\rho \underline{\mathbf{A}}_{R}
$$

where

$$
\rho=\sqrt{\frac{P_{R}}{\operatorname{Tr}\left[\left(\sum_{i=1}^{N} p_{i} \mathbf{h}_{i} \mathbf{h}_{i}^{H}+\sigma^{2} \mathbf{I}\right) \underline{\mathbf{A}}_{R}^{H} \underline{\mathbf{A}}_{R}\right]}}
$$

and the SNR at the MT $a_{k}$ is given by

$$
\gamma_{a_{k}}=\frac{\rho^{2} p_{b_{k}}}{\sigma^{2}+\rho^{2} \sigma_{R}^{2}\left\|\mathbf{f}_{a_{k}}\right\|^{2}}, \forall k
$$

where $\mathbf{F}=\left[\begin{array}{lllll}\mathbf{f}_{a_{1}} & \mathbf{f}_{b_{1}} & \ldots & \mathbf{f}_{a_{K}} & \mathbf{f}_{b_{K}}\end{array}\right]^{T}$.

\section{Block-Diagonalization for the TWRC}

The ZF structure in the previous section does not account for the fact that the RS should worry solely about eliminating inter-pair interference but not the intra-pair interference, since the individual users can take care of that themselves. A natural way to take this into consideration is to by adapting the BlockDiagonalization (BD) technique of [17] to the TWRC problem, which we do in the following.

If we define $\widetilde{\mathbf{H}}_{k}$ as

$$
\widetilde{\mathbf{H}}_{k}=\left[\begin{array}{llllll}
\mathbf{H}_{1} & \ldots & \mathbf{H}_{k-1} & \mathbf{H}_{k+1} & \ldots & \mathbf{H}_{K}
\end{array}\right],
$$

where $\mathbf{H}_{k}=\left[\mathbf{h}_{a_{k}} \mathbf{h}_{b_{k}}\right]$ is the uplink channel matrix of the $k$-th pair, then $\mathbf{F}_{k}$ should lie in the null space of $\widetilde{\mathbf{H}}_{k}$ so as to separate the uplink signals of each pair. Define $\tilde{L}_{k}=\operatorname{rank}\left(\widetilde{\mathbf{H}}_{k}\right) \leq N-$ 2 , as in [17]. We can define the singular value decomposition (SVD) of $\widetilde{\mathbf{H}}_{k}$ as follows:

$$
\widetilde{\mathbf{H}}_{k}=\left[\mathbf{U}_{\widetilde{\mathbf{H}}_{k}}^{(1)} \mathbf{U}_{\widetilde{\mathbf{H}}_{k}}^{(0)}\right] \Sigma_{\widetilde{\mathbf{H}}_{k}} \mathbf{V}_{\widetilde{\mathbf{H}}_{k}}^{H}
$$

where $\mathbf{U}_{\widetilde{\mathbf{H}}_{k}}^{(1)}$ holds the first $\tilde{L}_{k}$ left singular vectors, and $\mathbf{U}_{\widetilde{\mathbf{H}}_{k}}^{(0)}$ holds the last $M-\tilde{L}_{k}$ left singular vectors and forms an orthogonal basis for the null space of $\widetilde{\mathbf{H}}_{k}$. Define the following SVD for the channels of the $k$-th pair:

$$
\mathbf{U}_{\widetilde{\mathbf{H}}_{k}}^{(0) H} \mathbf{H}_{k}=\left[\mathbf{U}_{\mathbf{H}_{k}}^{(1)} \mathbf{U}_{\mathbf{H}_{k}}^{(0)}\right]\left[\begin{array}{cc}
\Sigma_{\mathbf{H}_{k}} & \mathbf{0} \\
\mathbf{0} & \mathbf{0}
\end{array}\right] \mathbf{V}_{\mathbf{H}_{k}}^{H}
$$

where $\Sigma_{\mathbf{H}_{k}}$ is $\bar{L}_{k} \times \bar{L}_{k}, \mathbf{U}_{\mathbf{H}_{k}}^{(1)}$ holds the first $\bar{L}_{k}$ left singular vectors. The product of $\mathbf{U}_{\mathbf{H}_{k}}^{(1) H}$ and $\mathbf{U}_{\widetilde{\mathbf{H}}_{k}}^{(0) H}$ produces an orthogonal basis of dimension $\bar{L}_{k}$.

Then, for the RS operation to have zero interference at each mobile pairs we select $\mathbf{F}_{k}$ as follows:

$$
\mathbf{F}_{k}=\mathbf{U}_{\mathbf{H}_{k}}^{(1) H} \mathbf{U}_{\widetilde{\mathbf{H}}_{k}}^{(0) H}=\left(\mathbf{U}_{\widetilde{\mathbf{H}}_{k}}^{(0)} \mathbf{U}_{\mathbf{H}_{k}}^{(1)}\right)^{H} \in \mathbb{C}^{\bar{L}_{k} \times M} .
$$

In second hop, the signal received at the $i$-th $\mathrm{MT}$ is given by (2). Let $\mathbf{G}=\left[\begin{array}{llll}\mathbf{g}_{1} & \mathbf{g}_{2} & \ldots & \mathbf{g}_{N}\end{array}\right]^{T} \in \mathbb{C}^{N \times M}$ for $\mathbf{g}_{k} \in \mathbb{C}^{M \times 1}$ be the overall channel matrix from the RS to the MTs in the second hop. If we define $\mathbf{G}_{k}=\left[\begin{array}{ll}\mathbf{g}_{a_{k}} & \mathbf{g}_{b_{k}}\end{array}\right]^{T} \in \mathbb{C}^{2 \times M}$, for $k=1,2, \ldots, K$, and let $\widetilde{\mathbf{G}}_{k}$ be equal to

$$
\widetilde{\mathbf{G}}_{k}=\left[\begin{array}{llllll}
\mathbf{G}_{1}^{T} & \ldots & \mathbf{G}_{k-1}^{T} & \mathbf{G}_{k+1}^{T} & \ldots & \mathbf{G}_{K}^{T}
\end{array}\right]^{T},
$$

then $\mathbf{M}_{k}$ should lie in the null space of $\widetilde{\mathbf{G}}_{k}$ for no inter-pair interference.

Define $\tilde{R}_{k}=\operatorname{rank}\left(\widetilde{\mathbf{G}}_{k}\right) \leq N-2$. We can express the SVD of $\widetilde{\mathbf{G}}_{k}$ as follows:

$$
\widetilde{\mathbf{G}}_{k}=\mathbf{U}_{\widetilde{\mathbf{G}}_{k}} \Sigma_{\widetilde{\mathbf{G}}_{k}}\left[\mathbf{V}_{\widetilde{\mathbf{G}}_{k}}^{(1)} \mathbf{V}_{\widetilde{\mathbf{G}}_{k}}^{(0)}\right]^{H}
$$

where $\mathbf{V}_{\widetilde{\mathbf{G}}_{k}}^{(1)}$ holds the first $\tilde{R}_{k}$ right singular vectors, and $\mathbf{V}_{\widetilde{\mathbf{G}}_{k}}^{(0)}$ holds the last $M-\tilde{R}_{k}$ right singular vectors and forms an orthogonal basis for the null space of $\widetilde{\mathbf{G}}_{k}$.

Define the following SVD for the channels of the $k$-th pair in the second hop:

$$
\mathbf{G}_{k} \mathbf{V}_{\widetilde{\mathbf{G}}_{k}}^{(0)}=\mathbf{U}_{\mathbf{G}_{k}}\left[\begin{array}{cc}
\Sigma_{\mathbf{G}_{k}} & \mathbf{0} \\
\mathbf{0} & \mathbf{0}
\end{array}\right]\left[\begin{array}{ll}
\mathbf{V}_{\mathbf{G}_{k}}^{(1)} & \mathbf{V}_{\mathbf{G}_{k}}^{(0)}
\end{array}\right]^{H}
$$

where $\Sigma_{\mathbf{G}_{k}}$ is $\bar{R}_{k} \times \bar{R}_{k}, \mathbf{V}_{\mathbf{G}_{k}}^{(1)}$ holds the first $\bar{R}_{k}$ right singular vectors. The product of $\mathbf{V}_{\mathbf{G}_{k}}^{(1)}$ and $\mathbf{V}_{\widetilde{\mathbf{G}}_{k}}^{(0)}$ produces an orthogonal basis of dimension $\bar{R}_{k}$. Then, for the RS operation we select $\mathbf{M}_{k}$ as follows:

$$
\mathbf{M}_{k}=\mathbf{V}_{\widetilde{\mathbf{G}}_{k}}^{(0)} \mathbf{V}_{\mathbf{G}_{k}}^{(1)} \in \mathbb{C}^{M \times \bar{R}_{k}} .
$$

After defining $\mathbf{F}_{k} \in \mathbb{C}^{\bar{L}_{k} \times M}$ and $\mathbf{M}_{k} \in \mathbb{C}^{M \times \bar{R}_{k}}$, we now give the structure for $\mathbf{D}_{k} \in \mathbb{C}^{\bar{R}_{k} \times \bar{L}_{k}}$. Define $\bar{Q}_{k}=$ $\min \left\{\bar{R}_{k}, \bar{L}_{k}\right\}$ and let the RS power scaling factor be $\rho$, then we assume the following structure for $\mathbf{D}_{k}$ :

$$
\mathbf{D}_{k}=\rho \underline{\mathbf{D}}_{k}=\rho\left[\begin{array}{cc}
\mathbf{I}_{\bar{Q}_{k} \times \bar{Q}_{k}} & \mathbf{0} \\
\mathbf{0} & \mathbf{0}
\end{array}\right] \in \mathbb{C}^{\bar{R}_{k} \times \bar{L}_{k}} .
$$

The RS transmit signal power is given by:

$$
\mathbb{E}\left[\mathbf{x}_{R}^{H} \mathbf{x}_{R}\right]=\operatorname{Tr}\left[\sum_{k=1}^{K} \mathbf{A}_{k} \mathbf{H}_{k} \mathbf{Q}_{k} \mathbf{H}_{k}^{H} \mathbf{A}_{k}^{H}+\sigma_{R}^{2} \mathbf{A}_{R} \mathbf{A}_{R}^{H}\right]
$$

where $\mathbf{Q}_{k}=\mathbb{E}\left[\mathbf{x}_{k} \mathbf{x}_{k}^{H}\right]=\operatorname{diag}\left(p_{a_{k}}, p_{b_{k}}\right)$. To meet the RS power constraint we need to select $\rho$ as

$$
\rho=\sqrt{\frac{P_{R}}{\operatorname{Tr}\left[\sum_{k=1}^{K} \underline{\mathbf{A}}_{k} \mathbf{H}_{k} \mathbf{Q}_{k} \mathbf{H}_{k}^{H} \underline{\mathbf{A}}_{k}^{H}+\sigma_{R}^{2} \underline{\mathbf{A}}_{R} \underline{\mathbf{A}}_{R}^{H}\right]}}
$$


where $\underline{\mathbf{A}}_{R}=\sum_{k=1}^{K} \mathbf{M}_{k} \underline{\mathbf{D}}_{k} \mathbf{F}_{k}$.

With the structures given by (17), (21) and (22) each pair of MTs is guaranteed to receive inter-pair interferencefree signals. After canceling the self-interference (using the knowledge of transmit signal), the received signal at MT $a_{k}$ and the corresponding received SINR are given by:

$$
\begin{gathered}
y_{a_{k}}=\mathbf{g}_{a_{k}}^{T} \mathbf{A}_{k} \mathbf{h}_{b_{k}} x_{b_{k}}+\mathbf{g}_{a_{k}}^{T} \mathbf{A}_{k} \mathbf{n}_{R}+n_{a_{k}}, \\
\gamma_{a_{k}}=\frac{p_{b_{k}}\left|\mathbf{g}_{a_{k}}^{T} \mathbf{A}_{k} \mathbf{h}_{b_{k}}\right|^{2}}{\sigma^{2}+\sigma_{R}^{2}\left\|\mathbf{g}_{a_{k}}^{T} \mathbf{A}_{k}\right\|^{2}}, \quad \forall k .
\end{gathered}
$$

IV. QUANTIZE-AND-FORWARD (QF) RELAYING

In this section, we consider QF relaying where the RS quantizes the signal vector it receives in the first phase and sends the corresponding bin index to the MTs in the second phase. Though sub-optimal, QF relaying is less complex than the CF relaying strategy in which the RS exploits the correlation between its received signal and the transmitted signal of each MT. We are lead to consider this scheme by the fact that it was shown in [3] that as the downlink channel quality improves, the system performance approaches the outer bound for the three-node relay channel. Here too, to avoid inter-pair interference, we resort to BD processing.

As each pair is only interested in part of the signal received at the RS, the latter first separates the received signals corresponding to each mobile pair by using the receive BD filter $\mathbf{F}$ given by (8) and (17), then proceeds to quantize each of the resulting signals independently. Thus, the processed received signal at the RS corresponding to the $k$-th pair is given by:

$$
\overline{\mathbf{y}}_{R_{k}}=\mathbf{F}_{k} \mathbf{y}_{R}=\mathbf{F}_{k} \mathbf{H}_{k} \mathbf{x}_{k}+\mathbf{F}_{k} \mathbf{n}_{R} .
$$

Moreover, since while decoding, each MT is able to subtract its own signal, we reduce the dimension of the source to be quantized from a two-dimensional vector, $\overline{\mathbf{y}}_{R_{k}}$ in 25), to a scalar, $z_{k}$, which is an appropriately selected linear combination of $\overline{\mathbf{y}}_{R_{k}}$ 's components and send that to both MTs in the corresponding pair. Thus:

$$
z_{k}=\mathbf{d}_{k}^{H} \overline{\mathbf{y}}_{R_{k}}=\mathbf{d}_{k}^{H} \mathbf{F}_{k} \mathbf{H}_{k} \mathbf{x}_{k}+\mathbf{d}_{k}^{H} \mathbf{F}_{k} \mathbf{n}_{R}
$$

where the combining vector $\mathbf{d}_{k}$ is selected so that the resulting pair sum rate is maximized; this is detailed below. In QF relaying, for each mobile pair, the RS wants to reliably forward the quantized signal $\hat{z}_{k}$ to the corresponding MTs in the pair where the quantized signal is selected according to the distribution $f\left(\hat{z}_{k} \mid z_{k}\right) \sim \mathcal{C N}\left(z_{k}, \sigma_{D_{k}}^{2}\right)$, where $\sigma_{D_{k}}^{2}$ is the noise variance due to the distortion in reconstructing $z_{k}$, i.e., $\hat{z}_{k}=z_{k}+n_{D_{k}}$ where $n_{D_{k}} \sim \mathcal{C N}\left(0, \sigma_{D_{k}}^{2}\right)$.

Now we need to reliably communicate from the RS to each mobile pair a single signal $s_{k}$ corresponding to that pair; signals corresponding to different pairs are independent. To do this, we use the multicast aware transmit beamforming scheme of [15] with a slight modification. The RS thus transmits

$$
\mathbf{x}_{R}=\left[\mathbf{M}_{1} \mathbf{b}_{1} \ldots \mathbf{M}_{K} \mathbf{b}_{K}\right]\left[\begin{array}{c}
s_{1} \\
\vdots \\
s_{K}
\end{array}\right]=\sum_{k=1}^{K} \mathbf{M}_{k} \mathbf{b}_{k} s_{k}
$$

where $\mathbf{M}_{k}$ was defined in the previous section (i.e. here too inter-pair interference is eliminated in the downlink) and $\mathbf{b}_{k}$ is selected so as to minimize distortion as will become clear in the following. With this structure the received signal at the MTs in the $k$-th pair is given by:

$\mathbf{y}_{k}=\left[\begin{array}{c}y_{a_{k}} \\ y_{b_{k}}\end{array}\right]=\mathbf{G}_{k} \mathbf{M}_{k} \mathbf{b}_{k} s_{k}+\mathbf{n}_{k}=\left[\begin{array}{c}\mathbf{g}_{a_{k}}^{T} \\ \mathbf{g}_{b_{k}}^{T}\end{array}\right] \mathbf{M}_{k} \mathbf{b}_{k} s_{k}+\mathbf{n}_{k}$.

For each pair, in order to be able to forward the quantized signal to both MTs, the following quantization rate constraint must be satisfied:

$$
I\left(\hat{z}_{k} ; z_{k}\right) \leq \min _{i \in\left\{a_{k}, b_{k}\right\}} I\left(s_{k} ; y_{i}\right)
$$

or equivalently, assuming Gaussian codebooks for transmission at the RS with $\mathbb{E}\left[\left|s_{k}\right|^{2}\right]=1$, the quantization variance should be lower bounded by

$$
\sigma_{D_{k}}^{2} \geq \frac{\sigma^{2} \mathbb{E}\left[\left|z_{k}\right|^{2}\right]}{\min _{i \in\left\{a_{k}, b_{k}\right\}}\left|\mathbf{g}_{i}^{T} \mathbf{M}_{k} \mathbf{b}_{k}\right|^{2}}
$$

where $\mathbb{E}\left[\left|z_{k}\right|^{2}\right]=P_{s}\left\|\mathbf{d}_{k}^{H} \mathbf{F}_{k} \mathbf{H}_{k}\right\|^{2}+\sigma_{R}^{2}\left\|\mathbf{d}_{k}^{H} \mathbf{F}_{k}\right\|^{2}$. Then, for the $a_{k}$-th MT we have the following SNR expression:

$$
\gamma_{a_{k}}=\frac{P_{s}\left\|\mathbf{d}_{k}^{H} \mathbf{F}_{k} \mathbf{h}_{b_{k}}\right\|^{2}}{\sigma_{R}^{2}\left\|\mathbf{d}_{k}^{H} \mathbf{F}_{k}\right\|^{2}+\sigma_{D_{k}}^{2}} .
$$

A similar equation holds for the SNR of $b_{k}$ by exchanging $a_{k}$ and $b_{k}$ in 31.

It was mentioned that $\mathbf{b}_{k}$ is selected so as to minimize distortion: referring back to (30), and assuming equal power allocation to each pair, it is thus the solution of the following optimization problem:

$$
\begin{gathered}
\operatorname{maximize}_{\mathbf{b}_{\mathbf{k}}} \min _{i \in\left\{a_{k}, b_{k}\right\}}\left|\mathbf{g}_{i}^{T} \mathbf{M}_{k} \mathbf{b}_{k}\right|^{2} \\
\text { such that }\left\|\mathbf{M}_{k} \mathbf{b}_{k}\right\|^{2}=\frac{P_{R}}{K}
\end{gathered}
$$

Also, $\mathbf{d}_{k}$ is selected so as to maximize the resulting sum rate of the $k$-th pair. Details of how this optimization problem and that in (32) are solved are omitted due to space limitations.

\section{Simulation Results}

Assume all MTs have equal power constraint $p_{i}=P_{s}, \forall i$ and $\sigma^{2}=\sigma_{R}^{2}=1$. We define the average SNR at the relay $S N R_{R S}=P_{s} / \sigma_{R}^{2}$ and at the MTs as $S N R_{M T}=P_{R} / \sigma^{2}$. The communication phases are divided into equivalent orthogonal time durations, i.e, all nodes are half-duplex. Reciprocal flat Rayleigh fading channels with unit variance for all of the channels on both phases are assumed. For the simulations, a $K=4$ pair ( $N=8 \mathrm{MTs})$ TWRC with $M=8$ antenna RS is considered.

We analyze and compare the average achievable sum-rates for the simple AF relaying scheme where the RS simply scales and forwards its received signal, the Tx-Rx ZF scheme, the Tx-Rx BD scheme and the proposed QF relaying scheme. As 


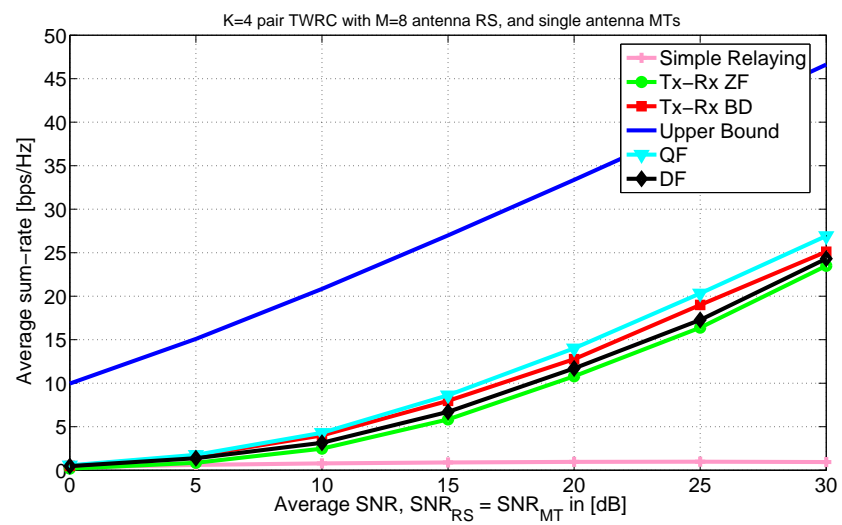

Fig. 2. Average sum-rate vs. $S N R_{R S}$ for $K=4$ pair TWRC with $M=8$ antenna RS and single antenna mobiles with $S N R_{M T}=S N R_{R S}$.

a benchmark we compare our proposed schemes to the $\mathrm{ZF}$ based DF relaying scheme from [15, Section 3.1].

Fig. 2 illustrates the achievable average sum-rate versus $S N R_{R S}$ with $S N R_{M T}=S N R_{R S}$. The proposed QF based relaying scheme achieves the best performance among the schemes considered. However, it is still far away from the MAC outer bound. Note that the performance of DF scheme is even inferior to the AF based Tx-Rx BD scheme because of using a $\mathrm{ZF}$ precoder on the downlink.

In Fig. 3, we plot the achievable average sum-rate versus $S N R_{M T}$ for $S N R_{R S}=10[\mathrm{~dB}]$. As the channel quality in the second phase, a bottleneck for the DF scheme, increases with $S N R_{M T}$, its achievable rate performance improves accordingly. However, the performance improvements in the $\mathrm{AF}$ and QF based schemes saturate with increasing $S N R_{M T}$ due to noise amplification and power consumption in channel inversion for the AF based schemes and not exploiting superposition coding in the second phase for the QF based scheme (assuming single user decoding capabilities for the MTs). But still for low $S N R_{M T}$, the QF is the best.

Note that the BD always outperforms the ZF scheme because ZF consumes additional degrees of freedom unnecessarily as it does not exploit the fact that each mobile has side information (its own signal). Moreover, the performance of simple relaying is heavily interference limited.

\section{CONCLUSION}

In a multi-pair TWRC, the main bottleneck on the system performance is the interference seen by each mobile from other communicating mobile pairs. This paper has tried to tackle this problem in the spatial domain by using multiple antennas at the relay node. Beamforming schemes for the relay using either AF or QF relaying strategies, were proposed. Moreover, for the QF relaying we proposed a signal combination scheme for each mobile pair at the relay station, which may be seen as an analog form of network coding. The sum-rate performance of the different schemes was illustrated via simulations and compared to the DF relaying strategy.

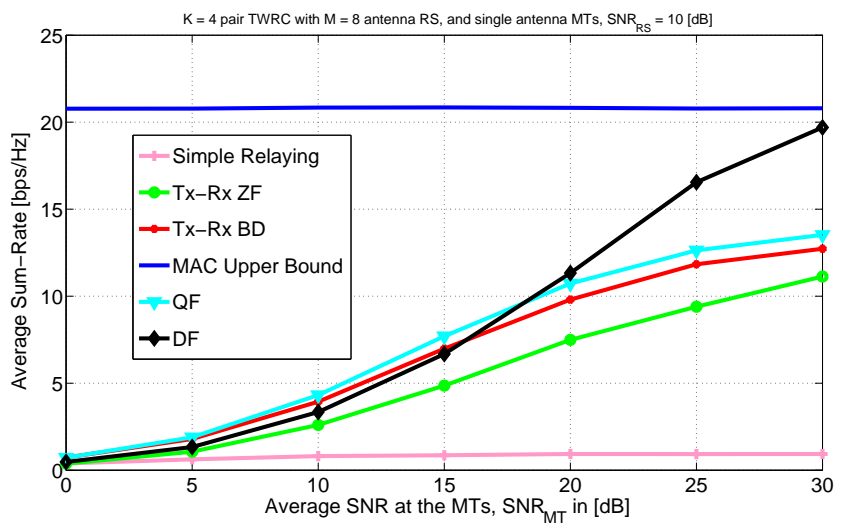

Fig. 3. Average sum-rate vs. $S N R_{M T}$ for $K=4$ pair TWRC with $M=8$ antenna RS and single antenna mobiles with $S N R_{R S}=10[\mathrm{~dB}]$.

\section{REFERENCES}

[1] T. Cover and A. El Gamal, "Capacity Theorems for the Relay Channel," IEEE Trans. on Info. Theory, vol. 25, no. 5, pp. 572-584, 1979.

[2] J. N. Laneman, D. N. C. Tse, and G.W. Wornell, "Cooperative Diversity in Wireless Networks: Efficient Protocols and Outage Behavior," IEEE Trans. on Info. Theory, vol. 50, no. 12, pp. 3062-3080, December 2004.

[3] G. Kramer, M. Gastpar, and P. Gupta, "Cooperative Strategies and Capacity Theorems for Relay Networks," IEEE Trans. on Info. Theory, vol. 51, no. 9, pp. 3037-3063, September 2005.

[4] B. Rankov and A. Wittneben, Spectral efficient protocols for half duplex fading relay channels, IEEE Journal on Selected Areas in Communications, vol. 25, no. 2, pp. 379389, February 2007.

[5] R. Knopp, "Two-way wireless communication via a relay station," GDRISIS meeting, March 2007.

[6] P. Popovski and H. Yomo, "Bi-directional amplification of throughput in a wireless multi-hop network, in proc. of the IEEE VTC-Spring'06, pp. $588-593$

[7] T. J. Oechtering, C. Schnurr, I. Bjelakovic, and H. Boche, Achievable rate region of a two phase bidirectional relay channel, in proc. of the 41st Annual Conf. on Info. Sciences and Systems, CISS07, March 2007.

[8] S. Katti, S. Gollakota, and D. Katabi, "Embracing Wireless Interference: Analog Network Coding," in proc. of the ACM SIGCOMM, 2007.

[9] C. Schnurr, S. Stanczak, and T. J. Oechtering, Coding theorems for the restricted half-duplex two-way relay channel with joint decoding, in proc. of the IEEE Int'l Symp. on Info. Theory, Canada, July 2008.

[10] S. Zhang, S. Liew, and P. P. Lam, "Physical-Layer Network Coding," in Proceedings of the ACM MOBICOM, 2006.

[11] S. J. Kim, P. Mitran, and V. Tarokh, Performance bounds for bidirectional coded cooperation protocols, in proc. of the 27th Int'l conf. on Distributed Computing Systems Workshops, ICDCSW07, June 2007.

[12] T. Unger and A. Klein, "Duplex schemes in multiple-antenna two-hop relaying," EURASIP JASP, (special issue: "Multihop-Based Cellular Networks"), vol. 8, issue 2, pp. 1-14, Jan. 2008.

[13] R. Zhang, Y. C. Liang, C. C. Chai, and S. Cui, "Optimal Beamforming for Two-Way Multi-Antenna Relay Channel with Analogue Network Coding, “ http://arxiv.org/abs/0808.0075v2

[14] C. Esli and A. Wittneben, "One- and Two-Way Decode-and-Forward Relaying for Wireless Multiuser MIMO Networks," IEEE Global Communications Conference (IEEE Globecom 08), Nov. 2008.

[15] A. U. T. Amah, A. Klein, Y. C. B. Silva, A. Fernekess, "Multi-Group Multicast Beamforming For Multi-User Two-Way Relaying," Int'l ITG Workshop on Smart Antennas WSA, Berlin, Germany, February 2009.

[16] M. Chen and A. Yener,"Multiuser two-way relaying for interference limited systems, " in Proc. IEEE International Conference on Communications, pp.3883-3887, Beijing, May 2008.

[17] Q. H. Spencer, A. L. Swindlehurst, and M. Haardt, "Zero-forcing methods for downlink spatial multiplexing in multiuser MIMO channels," IEEE Trans. Signal Processing, vol. 52, no. 2, pp. 461-471, 2004. 